\title{
La sinodalità nell'ecclesiologia di Papa Francesco
}

\author{
Synodality in the ecclesiology of Pope Francis
}

Riassunto: La sinodalità, come dimensione costitutiva della Chiesa, ci offre la cornice interpretativa più adeguata per comprendere lo stesso ministero gerarchico. San Giovanni Crisostomo ha detto che «Chiesa e Sinodo sono sinonimi», perché all'interno della Chiesa nessuno può essere "elevato" al di sopra degli altri. Al contrario, nella Chiesa è necessario che qualcuno "si abbassi" per mettersi al servizio dei fratelli lungo il cammino. Una Chiesa sinodale è una Chiesa dell'ascolto, nella consapevolezza che ascoltare «è più che sentire». '̇ un ascolto reciproco in cui ciascuno ha qualcosa da imparare. Popolo fedele, Collegio episcopale, Vescovo di Roma: l'uno in ascolto degli altri; e tutti in ascolto dello Spirito Santo, lo «Spirito della verità» $(G v$ 14,17), per conoscere ciò che Egli «dice alle Chiese» (Ap 2,7).

Parole-chiave: Sinodalità; sinodo dei vescovi; collegialità; Chiesa; diritto canonico.

\section{Manlio Miele}

Abstract: Synodality is a constitutive element of the Church and offers us the most appropriate interpretive framework for understanding the hierarchical ministry itself. Saint John Chrysostom says that "Church and Synod are synonymous": it means that, within the Church, no one can be "raised up" higher than others. On the contrary, in the Church, it is necessary that each person "lower" himself or herself, so as to serve our brothers and sisters along the way. A synodal Church is a Church which listens, which realizes that listening "is more than simply hearing". It is a mutual listening in which everyone has something to learn. The faithful people, the college of bishops, the Bishop of Rome: all listening to each other, and all listening to the Holy Spirit, the "Spirit of truth" (Jn 14:17), in order to know what he "says to the Churches" (Rev 2:7).

Keywords: Synodality; Synod of Bishops; collegiality; Church; canon law.

\footnotetext{
${ }^{1}$ Professore Ordinario di Diritto canonico ed ecclesiastico, Università degli Studi di Padova.
} 


\section{Introduzione}

Mentre era in corso il Concilio Vaticano II, Paolo VI, aprendone la quarta sessione il 14 settembre 1965, annunciava la creazione di un «sinodo di vescovi» ${ }^{2}$.

La decisione non era inattesa dal momento che il numero 5 del decreto conciliare sulla missione pastorale dei vescovi, Christus Dominus, ne aveva delineato l'essenza ${ }^{3}$. Esso doveva essere composto da alcuni vescovi e doveva essere convocato dal pontefice per trattare delle necessità della Chiesa e, quando l'avesse ritenuto opportuno, per averne collaborazione e consiglio.

Paolo VI, aprendo la quarta sessione dell'assise conciliare, esprimeva anche l'idea che il sinodo dovesse essere di giovamento, in modo particolare, al lavoro quotidiano della Curia romana ${ }^{4}$.

Il 15 settembre veniva pubblicato il motu proprio Apostolica sollicitudo che creava il nuovo istituto ${ }^{5}$.

Del sinodo venivano configurate tre tipologie di assemblea:

- generale, alla quale avrebbero partecipato i Patriarchi, gli Arcivescovi Maggiori ed i Metropoliti fuori dei Patriarcati delle Chiese Cattoliche di rito orientale; i Vescovi eletti dalle

\footnotetext{
${ }^{2}$ Cfr. Faggioli, Il vescovo e il Concilio. Modello episcopale e aggiornamento al Vaticano II, cit., pp. 389-438. Studi monografici: AA. VV., Il sinodo dei vescovi. Natura, metodo, prospettive, a cura di J. Tomko, Roma, Libreria editrice vaticana, 1985; G.P. Milano, Il sinodo dei vescovi, cit.; J.I. Arrieta, El Sínodo de los Obispos, Pamplona, 1987; M.C. Bravi, Il sinodo dei vescovi. Istituzione, fini e natura, Roma, 1995; A. Indelicato, Il sinodo dei vescovi. La collegialità sospesa (1965-1985), Bologna, 2008.

3 «Una più efficace collaborazione al supremo pastore della Chiesa la possono prestare, nei modi dallo stesso romano Pontefice stabiliti o da stabilirsi, i vescovi scelti da diverse regioni del mondo, riuniti nel consiglio propriamente chiamato Sinodo dei vescovi. Tale Sinodo, rappresentando tutto l'episcopato cattolico, è un segno che tutti i vescovi sono partecipi in gerarchica comunione della sollecitudine della Chiesa universale».

${ }^{4}$ Il discorso si trova in AAS 57 (1965), 794-805; il passo citato è a pagina 804.

${ }^{5}$ Paolo VI, motu proprio Apostolica sollicitudo, 15 settembre 1965, in AAS 57 (1965), 775-780 (Enchiridion Vaticanum [= EV], 2, 472-481). 
singole conferenze Episcopali Nazionali; i Vescovi eletti dalle Conferenze Episcopali di più Nazioni, costituiti cioè per quelle Nazioni che non hanno una conferenza propria; dieci religiosi rappresentanti gli Istituti Religiosi Clericali, eletti dall'Unione Romana dei Superiori Generali nonché i Cardinali preposti alla direzione dei Dicasteri della Curia Romana (art. V);

- straordinaria, con i Patriarchi, gli Arcivescovi Maggiori ed i Metropoliti fuori dei Patriarcati delle Chiese Cattoliche di rito orientale; i Presidenti delle Conferenze Episcopali Nazionali; i Presidenti delle Conferenze Episcopali di più Nazioni, costituite per quelle Nazioni che non hanno una Conferenza Episcopale propria; tre religiosi rappresentanti gli Istituti Religiosi Clericali, eletti dall'Unione Romana dei Superiori Generali nonché i Cardinali preposti alla direzione dei Dicasteri della Curia Romana (art. VI);

- speciale, che avrebbe compreso i Patriarchi, gli Arcivescovi Maggiori e i Metropoliti fuori dei Patriarcati delle Chiese Cattoliche di rito orientale, come pure coloro che rappresentano sia le Conferenze Episcopali di una o più Nazioni sia gli Istituti Religiosi purché tutti appartengano alle regioni per la quali il Sinodo dei Vescovi è stato convocato (art. VII).

\section{Rappresentatività e rappresentanza}

Dalle citate disposizioni potevano desumersi i lineamenti generali e basilari del nuovo istituto: 1) il sinodo era composto solo da alcuni vescovi cattolici, di provenienza universale e variamente designati, ma tutti legittimati da un intervento del pontefice ${ }^{6}$;2) la sua competenza ordinaria era di natura consultiva; 3) esso doveva

\footnotetext{
${ }^{6} \mathrm{Nel}$ caso dei vescovi designati dalle Conferenze episcopali, «eletti» secondo gli articoli V e VIII del Motuproprio ed il can. 346, $§ 1$, si è giustamente intravista più una forma di presentazione che non di elezione canonica. Cfr. J.I. Arrieta, Diritto dell'organizzazione ecclesiastica, Milano, 1997, pp. 273-274.
} 
considerarsi come posto in rappresentanza di tutto l'episcopato cattolico («partes agens totius catholici Episcopatus») ${ }^{7}$.

Quest'ultimo aspetto sarebbe stato oggetto di vivace dibattito in dottrina anche, probabilmente, per una certa disinvoltura nell'usare come identici i concetti di rappresentanza e di rappresentatività senza tenere in adeguato conto la diversa natura dei rapporti motivati in termini di democrazia rappresentativa rispetto a quelli fondati su base sacramentale, sulla cui ontologia tanto insistette Eugenio Corecco nel

${ }^{7}$ Il Motu proprio articolava le caratteristiche del sinodo dicendo che esso era regolato così da essere: «a) una istituzione ecclesiastica centrale; b) rappresentante tutto l'Episcopato cattolico; c) perpetua per sua natura; d) quanto alla sua struttura, svolgente i suoi compiti in modo temporaneo ed occasionale» (I).

${ }^{8}$ Sul rilievo della distinzione, nell'ordinamento ecclesiale, si veda la relazione (9 marzo 2012) di P. Gherri in http://gherripaolo.eu/lucera_discernimento.pdf, nonché L. Musselli, Forme ed Istituti di rappresentanza canonica, in Paolo Gherri (ed.), Responsabilità ecclesiale, corresponsabilità e rappresentanza (Atti della quarta giornata canonistica interdisciplinare), Roma, 2010, pp. 110-112; M. Miele, Dalla sinodalità alla collegialità nella codificazione latina, Padova, 2004, p. 199, nota 135. Sul rapporto tra il ministero e il fenomeno della rappresentanzarappresentazione: J.-M. Tillard, Chiesa di chiese. L'ecclesiologia di comunione, trad. it., Brescia, 1989, pp. 223-232. Libero Gerosa, a proposito del voto nei consigli ecclesiali di ogni livello ecclesiale, non distingue tra rappresentanza e rappresentatività, ma tra rappresentanza e testimonianza della fede, giacché «il concetto di "rappresentanza"... ha un significato diverso nella struttura ecclesiale retta dal principio della "communio" e conosciuto per fede, perché quest'ultima non può essere rappresentata ma solo testimoniata». Conseguentemente i membri di assemblee ecclesiali «anche quando sono eletti con criteri "rappresentativi" o democratici, non sono dei "rappresentanti" di tipo parlamentare ma dei fedeli scelti per testimoniare la loro fede ed aiutare secondo "scienza e competenza" (can. $212 \S$ 3) il fedele che - in forza del sacramento dell'ordine e della "missio canonica" - è investito d'autorità nella comunità cristiana in questione» (L. Gerosa, Inveramenti istituzionali delle nozioni conciliari di «collegialità»e «partecipazione», cit., p. 81). Rimane il fatto che la «testimonianza» non si realizza in modo astorico e disgiunto dall'appartenenza a diversa «lingua, popolo e nazione» e a diversi ambiti culturali e sociali; in tal senso un'assemblea ecclesiale può realizzare una sua rappresentatività senza potersi definire "rappresentante" dei fedeli che non ne fanno parte. 
parlare sia della sinodalità ecclesiale che degli organismi partecipativi espressione della stessa ${ }^{9}$.

Anche per porre fine a simili fraintendimenti, il Codex del 1983 evitava di riproporre la formula del sinodo come istituto «partes agens totius catholici Episcopatus $\rangle^{10}$. Il che, forse, non era neppure necessario, laddove si avvertisse come nel Motuproprio del 1965 il sinodo era posto in rapporto all'«episcopato cattolico» e non al «Collegio episcopale» ${ }^{11}$.

\footnotetext{
${ }^{9}$ Molti degli scritti di Corecco, anche su questo tema, sono raccolti nei volumi: E. Corecco, Ordinatio Fidei, Schriften zum kanonischen Recht, a cura di L. Gerosa e L. Müller, Paderborn, 1994; E. Corecco, «Ius et communio». Scritti di diritto canonico, a cura di G. Borgonovo e A. Cattaneo, Casale Monferrato, 1997, 2 v. Per un interessante approfondimento sugli scritti di Corecco relativamente al tema della sinodalità, cfr. C. Fantappiè, Chiesa e sinodalità. Per un confronto con Eugenio Corecco, «Ephemerides iuris canonici» 58 (2/2018), 461-478.

${ }^{10}$ La Pontificia Commissione per la revisione del Codex, sul punto, rispose alla Segreteria generale del sinodo dei vescovi, con propria lettera del 20 settembre 1983 e allegando un parere di W. Onclin: «Come si disse nella discussione su questa definizione, se il Sinodo dei Vescovi rappresentasse veramente tutti i Vescovi, sarebbe come il Concilio ecumenico e dovrebbe avere voto deliberativo e i suoi atti sarebbero atti collegiali. Ma questo non corrisponde alla realtà. Il Sinodo dei Vescovi non si può considerare come un Collegio di tutti i Vescovi; sarebbe un Concilio ecumenico»: Il sinodo dei vescovi. Natura, metodo, prospettive, cit., p. 180. ${ }^{11}$ Sulla facilità con cui talvolta si è posta una relazione (giuridica) diretta tra sinodo e Collegio episcopale, si vedano i cenni in A. Remossi, Il concetto di rappresentatività nell'ordinamento canonico, in Periodica de re canonica, 2005, 94, pp. 604-607. Il rapporto tra l'uno e l'altro investe anche un problema di autorità ex iure divino, così come evidenziato da Legrand, per il quale il sinodo «potrebbe facilmente diventare un organismo più rappresentativo dell'episcopato senza pretendere che le sue raccomandazioni abbiano l'autorità del collegio universale»: Legrand, Le riforme di Francesco, cit., p. 423. V. De Paolis, in una conferenza tenuta a Madrid il 26 novembre 2014, dopo la pubblicazione della Relatio finalis del sinodo straordinario del 2014, si sarebbe chiesto se sia «competenza di un sinodo dei vescovi trattare una questione come questa: il valore della dottrina e della disciplina vigente nella Chiesa, che si sono formate nel corso dei secoli e sono sancite con interventi del magistero supremo della Chiesa», almeno secondo quanto riportato in http://chiesa.espresso.repubblica.it/articolo/1350935.
} 
Papa Francesco, nella costituzione apostolica Episcopalis communio con cui ha riformato il Sinodo dei Vescovi, ha evitato di riproporre l'art. 1 del Motuproprio Apostolica sollicitudo di Paolo VI, omettendo, pertanto, ogni riferimento espresso al fatto che il Sinodo dei vescovi sia «partes agens totius catholici Episcopatus» e per il resto rinviando al can. 346 CIC quanto ai membri e agli altri partecipanti delle Assemblee.

Data la sua composizione, si ritiene di poter affermare che il sinodo è rappresentativo dell'episcopato cattolico senza, per questo, essere rappresentante della totalità dei vescovi appartenenti al Collegio episcopale. Del resto, il Codex del 1983 non ha esitato ad utilizzare il linguaggio della rappresentanza, in modo esplicito, nel caso di un altro istituto sconosciuto al Codex del 1917, il Consiglio presbiterale, definito non solo come senato del vescovo, ma anche come gruppo di sacerdoti «presbyterium repraesentans» (can. 495, $\S$ $1)^{12}$. Le discussioni conciliari sulla strutturazione di questo organo collegiale sono state ricostruite, notandosi come poi, a proposito del Consiglio presbiterale, fosse emersa da un lato una «repraesentatio per mimèsi o per imitazione», così che il Consiglio riproducesse «in piccolo la varietà delle situazioni del presbiterio»; dall'altro, come la stessa repraesentatio del presbiterio spettasse «a tutto il consiglio presbiterale non ai singoli membri» ${ }^{13}$.

Se è vero che nel Codex del 1983 come pure nella costituzione apostolica Episcopalis communio del sinodo episcopale non viene formalmente ribadito l'aspetto di rappresentanza, non c'è dubbio che la rinnovata esigenza che i suoi componenti, nell'ambito di

\footnotetext{
${ }^{12}$ Il Consiglio presbiterale eredita il titolo di senatus che nel Codex del 1917 era attribuito al Capitolo cattedrale (can. 391, § 1). Viceversa, nello stesso Codex, non vi è alcun accenno ad una rappresentanza del Capitolo rispetto al clero diocesano.

${ }^{13}$ A. Longhitano, «Repraesentatio» e partecipazione nell'ordinamento canonico, in Repraesentatio. Sinodalità ecclesiale e integrazione politica, Atti del Convegno di studi organizzato dallo Studio Teologico San Paolo e dalla Facoltà di Giurisprudenza dell'Università degli Studi di Catania, Catania 21-22 aprile 2005, a cura di A. Longhitano, Firenze, 2007, pp. 181-183.
} 
un'assemblea generale, siano scelti da tutte le regioni del mondo (can. 342 CIC), configuri un metodo per garantire una rappresentatività geografica, e quindi culturale, a livello mondiale; anche i vescovi padri del sinodo riunito in assemblea generale quindi, in quanto scelti effettivamente da tutto il mondo, rappresentano per imitazione tutto l'episcopato cattolico, episcopato che, come la Chiesa, è «di ogni lingua, popolo e nazione» e tenendo presente che il legame di ogni Chiesa particolare ad un singolo mondo culturale è elemento «costitutivo della sua Cattolicità» ${ }^{14}$. E tale rappresentatività è particolarmente rafforzata per quei vescovi che vengono scelti tramite l'elezione in seno alle Conferenze episcopali. Nelle assemblee generali ordinarie questi costituiscono il maggior numero dei padri sinodali e durante la celebrazione sinodale essi dovrebbero esprimere l'opinione emersa in seno alla discussione sui temi sinodali previamente effettuata dalla Conferenza episcopale di provenienza ${ }^{15}$. Questa disposizione poteva far pensare ad un vero e proprio meccanismo di delega-rappresentanza tra le Conferenze (deleganti) ed i vescovi (delegati); tuttavia, in relazione ad essa, già nel 1977 alcune Note esplicative della Segreteria generale chiedevano che i vescovi designati al sinodo esponessero i pareri sia della maggioranza che della minoranza della propria Conferenza ${ }^{16}$, con ciò riecheggiandosi l'antica dialettica canonica tra maioritas et sanioritas. Il problema di riferire al sinodo le opinioni diverse, presenti in seno alla Conferenza

\footnotetext{
${ }^{14}$ H.-M. Legrand, Synodes et conseils de l'après-concile, in Nouvelle revue theologique, 1976, 98, p. 196.

${ }^{15}$ Così dispone l'art. 23 dell' Ordo Synodi Episcoporum, nell'ultima redazione approvata da Benedetto XVI il 29 settembre 2006: AAS 98 (2006) 755-779 (EV, 23, 1526-1577).

16 «Questo articolo del Regolamento del Sinodo prescrive che i Delegati delle singole Conferenze Episcopali manifestino la posizione dei propri fratelli nell'Episcopato, a riguardo degli argomenti che il Sommo Pontefice ha stabilito di trattare nella convocazione del Sinodo. Per poter dare, a proposito del medesimo voto, una conoscenza il più possibile accurata, i delegati si adoperino affinché, dopo aver esposto il parere della maggioranza, presentino anche l'opinione della minoranza della propria Conferenza». V. J.I. Arrieta, Il sistema dell'organizzazione ecclesiastica, I ed., Roma, 2000, p. 59.
} 
episcopale delegante, non si pone infatti quando essa sia unanime sulle stesse ${ }^{17}$. Anche per ciò si è sottolineato l'aspetto di rappresentatività morale, e non «giuridico-formale», del sinodo ${ }^{18}$.

Alla luce di tutto ciò, si possono comprendere le parole di Giovanni Paolo II circa le dinamiche del sinodo episcopale: «Ogni Assemblea Generale del Sinodo dei Vescovi è una forte esperienza ecclesiale, anche se nelle modalità delle sue procedure rimane sempre perfettibile. I Vescovi riuniti nel Sinodo rappresentano anzitutto le proprie Chiese, ma tengono presenti anche i contributi delle Conferenze episcopali dalle quali sono designati e dei cui pareri circa le questioni da trattare si fanno portatori. Essi esprimono così il voto del Corpo gerarchico della Chiesa e, in qualche modo, quello del popolo cristiano, del quale sono i pastori. Il Sinodo è un evento in cui si rende particolarmente evidente che il Successore di Pietro, nell'adempimento del suo ufficio, è sempre congiunto nella comunione con gli altri Vescovi e con tutta la Chiesa. «Spetta al Sinodo dei Vescovi - stabilisce al riguardo il Codice di Diritto Canonico discutere sulle questioni da trattare ed esprimere propri voti, non però

\footnotetext{
${ }^{17}$ Per analogia si deve qui ricordare che - per ciò che riguarda l'esercizio magisteriale da parte delle Conferenze episcopali -, laddove una dichiarazione della Conferenza non sia adottata all'unanimità, essa può reputarsi atto di magistero autentico solo se ottenga la recognitio della Sede apostolica romana, dopo essere stata approvata dalla maggioranza qualificata (dei due terzi) dei vescovi dotati di voto deliberativo (Giovanni Paolo II, motu proprio Apostolos Suos, 21 maggio 1998, n. 22, in AAS 90 [1998] 641-658 e in EV, 17, 518-555). Si è osservato in proposito come «il motu proprio, nel momento in cui richiede l'unanimità ai fini della validità ed efficacia degli atti di magistero autentico di una conferenza di vescovi, pone anch'esso un limite alla volontà della maggioranza, ma per garantire un oggetto specifico ben diverso dall'interesse del singolo, e cioè per garantire e promuovere la retta comprensione della dottrina della Chiesa nel suo sviluppo dogmatico»: A. Bettetini, Formazione della volontà collegiale, principio democratico e verità nel diritto della Chiesa, in Repraesentatio. Sinodalità ecclesiale e integrazione politica, cit., p. 165.

${ }^{18}$ Arrieta, Diritto dell'organizzazione ecclesiastica, cit., pp. 267-268. Su questo punto si veda J. Ratzinger, Chiesa, ecumenismo e politica. Nuovi saggi di ecclesiologia, trad. it., Milano, 1987, pp. 59-61; cfr. anche G. Canobbio, Libertà di parola e sinodalità tra diritto e responsabilità, Apostolicam actuositatem, Roma 2017.
} 
dirimerle ed emanare decreti su di esse, a meno che in casi determinati il Romano Pontefice, cui spetta in questo caso ratificare le decisioni del Sinodo, non abbia concesso potestà deliberativa». Il fatto che il Sinodo abbia normalmente una funzione solo consultiva non ne diminuisce l'importanza. Nella Chiesa, infatti, il fine di qualsiasi organo collegiale, consultivo o deliberativo che sia, è sempre la ricerca della verità o del bene della Chiesa. Quando poi si tratta della verifica della medesima fede, il consensus Ecclesiae non è dato dal computo dei voti, ma è frutto dell'azione dello Spirito, anima dell'unica Chiesa di Cristo. Proprio perché il Sinodo è al servizio della verità e della Chiesa, come espressione della vera corresponsabilità da parte di tutto l'episcopato in unione con il suo Capo riguardo al bene della Chiesa, nel dare il voto o consultivo o deliberativo i Vescovi, insieme agli altri membri del Sinodo, esprimono comunque la partecipazione al governo della Chiesa universale. Come il mio predecessore di v. m. Paolo VI, anche io ho sempre fatto tesoro delle proposte e dei pareri espressi dai Padri sinodali, facendoli entrare nel processo di elaborazione del documento che raccoglie i risultati del Sinodo, e che proprio per questo amo qualificare come «post-sinodale» ${ }^{19}$.

Nello stesso senso, del resto, si è espresso Papa Francesco nel già citato Discorso in occasione della Commemorazione del 50.mo anniversario dell'Istituzione del Sinodo dei Vescovi del 17 ottobre 2015. Ha infatti affermato l'attuale Pontefice che «una Chiesa sinodale è una Chiesa dell'ascolto, nella consapevolezza che ascoltare «è più che sentire». È un ascolto reciproco in cui ciascuno ha qualcosa da imparare. Popolo fedele, Collegio episcopale, Vescovo di Roma: l'uno in ascolto degli altri; e tutti in ascolto dello Spirito Santo, lo «Spirito della verità» $(\mathrm{Gv} 14,17)$, per conoscere ciò che Egli «dice alle Chiese» (Ap 2,7). Il Sinodo dei Vescovi è il punto di convergenza di questo dinamismo di ascolto condotto a tutti i livelli della vita della Chiesa. Il cammino sinodale inizia ascoltando il Popolo, che «pure

\footnotetext{
${ }^{19}$ Giovanni Paolo II, esortazione apostolica postsinodale Pastores gregis, 16 ottobre 2003, in AAS 96 (2004) 825-924 e in EV, 22, 506-741. Quello citato è il numero 58.
} 
partecipa alla funzione profetica di Cristo», secondo un principio caro alla Chiesa del primo millennio: "Quod omnes tangit ab omnibus tractari debet». Il cammino del Sinodo prosegue ascoltando i Pastori. Attraverso i Padri sinodali, i Vescovi agiscono come autentici custodi, interpreti e testimoni della fede di tutta la Chiesa, che devono saper attentamente distinguere dai flussi spesso mutevoli dell'opinione pubblica. Alla vigilia del Sinodo dello scorso anno affermavo: «Dallo Spirito Santo per i Padri sinodali chiediamo, innanzitutto, il dono dell'ascolto: ascolto di Dio, fino a sentire con Lui il grido del Popolo; ascolto del Popolo, fino a respirarvi la volontà a cui Dio ci chiama». Infine, il cammino sinodale culmina nell'ascolto del Vescovo di Roma, chiamato a pronunciarsi come «Pastore e Dottore di tutti i cristiani»: non a partire dalle sue personali convinzioni, ma come supremo testimone della fides totius Ecclesiae, «garante dell'ubbidienza e della conformità della Chiesa alla volontà di Dio, al Vangelo di Cristo e alla Tradizione della Chiesa» ${ }^{20}$.

\section{Interazione tra il sinodo ed il Collegio dei cardinali}

L'esperienza del sinodo iniziò nel 1967 e si sviluppò in modo costante così che, fino al 2014, in quarantasette anni si ebbero - tra assemblee ordinarie, straordinarie e speciali -, ventisei sinodi, quasi uno ogni due anni ${ }^{21}$. In nessuno di questi sinodi il pontefice si avvalse della possibilità, prevista sia dal Motuproprio istitutivo che dal can. 343 del Codex, di delegare al sinodo potestà deliberativa. Vero è che il papa pubblicò numerose esortazioni apostoliche postsinodali, composte più sulla base delle proposizioni presentate dal sinodo che non dei singoli interventi svolti nell'aula sinodale.

\footnotetext{
${ }^{20}$ Francesco, Discorso in occasione della Commemorazione del 50.mo anniversario dell'Istituzione del Sinodo dei Vescovi, 17 ottobre 2015, cit.

${ }^{21} \mathrm{Si}$ veda N. Eterović (ed.), Il sinodo dei vescovi. 40 anni di storia. 1965-2005, Città di Castello (PG), 2005; Id., Sinodi continentali. I consigli speciali del sinodo dei vescovi, Città del Vaticano, 2013; Id., Joseph Ratzinger. Benedetto XVI e il sinodo dei vescovi, Città del Vaticano, 2014.
} 
Nel contempo andava sviluppandosi nella prassi, sempre a livello centrale, un nuovo coinvolgimento del Collegio dei cardinali, attraverso la convocazione a Roma, da parte del papa, di riunioni plenarie dei cardinali. In un certo senso, queste «plenari» erano presentate come munite di una fisionomia distinta dal tradizionale istituto dei concistori; ciò sembrerebbe trasparire chiaro fin dalla prima riunione, voluta e celebrata da Giovanni Paolo II tra il 5 e il 9 novembre 1979. Nel discorso inaugurale il Papa, già allora, rivelava come la richiesta che i cardinali venissero tutti convocati a Roma, al di fuori dell'evento della sede vacante, fosse emersa durante le congregazioni preparatorie dei conclavi del 1978. In quell'occasione il Papa tracciava un certo statuto di tali plenarie. I cardinali non solo prestavano il loro aiuto al papa, «in modo continuo e costante», in quanto appartenenti alla Curia romana, ma, tutti, condividevano con lui la «comune sollecitudine per la Chiesa» in forza del loro inserimento nel clero romano. Se questa idea era consolidata nel Codex allora vigente ${ }^{22}$, il Papa aggiungeva, come ulteriore fondamento alle plenarie cardinalizie, la «collegialità vescovile e pastorale, che è in vigore da oltre mille anni $\rangle^{23}$. Il riferimento del Collegio cardinalizio al principio di collegialità episcopale era estraneo sia al Codex del 1917 che ai documenti del Vaticano II, i quali, del resto, questo Collegio non l'avevano mai nominato. Esso, però, corrispondeva ad alcune, più o meno percettibili, trasformazioni disciplinari che lo avevano riguardato fin dalla vigilia del Concilio oltre che ad alcuni interventi dottrinali ${ }^{24}$.

Per le trasformazioni nella disciplina va ricordato anzitutto come, alla vigilia del Concilio, Giovanni XXIII avesse stabilito che tutti

\footnotetext{
${ }^{22}$ Can. 230 Codex 1917.

${ }^{23}$ Giovanni Paolo II, Discorso ai partecipanti alla riunione plenaria del Sacro Collegio dei cardinali, 5 novembre 1979, in Insegnamenti di Giovanni Paolo II, II, 2, 1979, 1060-1069 e in AAS 71 (1979), 1447-1457.

${ }^{24}$ Per il dibattito sull'alternativa tra «riforma del collegio cardinalizio o creazione di un "Consilium episcoporum centrale"»; cfr. Faggioli, Il vescovo e il Concilio. Modello episcopale e aggiornamento al Vaticano II, cit., pp. 403-406.
} 
i cardinali venissero consacrati vescovi ${ }^{25}$. Paolo VI poi, prima della fine del Concilio, forniva una nuova legittimazione alla presenza dei patriarchi orientali nel Collegio dei cardinali stabilendo la recisione del loro legame con la Chiesa romana, significato nella titolarità di una diocesi suburbicaria o nell'appartenenza al clero romano ${ }^{26}$.

Per l'elaborazione dottrinale ${ }^{27}$, anzitutto sul piano della ricostruzione e dell'interpretazione storica, va ricordato certamente uno studio di Congar, pubblicato verso la fine del Concilio e nel quale egli parlava di una progressiva «confisca», da parte dell'«ideologia del collegio dei cardinali», della teologia della collegialità episcopale ${ }^{28}$. Qualche anno dopo, Giuseppe Alberigo pubblicava un volume dedicato all'ecclesiologia tra l'XI e il XIV secolo e nel quale si accentuava l'osmosi, se non la sostituzione, attuatasi nel secondo millennio, tra lo statuto del cardinalato e la tradizione sinodale riferita al Collegio apostolico e quindi all'episcopato ${ }^{29}$. Questo lavoro di Alberigo, in chiusura, metteva in rilievo il pensiero dell'Autore sul venir meno di qualunque base dottrinale alla persistenza del collegio cardinalizio e sul

${ }^{25}$ Giovanni XXIII, motu proprio Cum gravissima, 15 aprile 1962, in AAS 54 (1962) 256-258. Nel discorso dopo il rito di consacrazione, il successivo 19 aprile 1962, il Papa non accenna in alcun modo alla valenza che l'atto poteva avere nei confronti del Collegio episcopale, convocato a Concilio, e sottolinea la «parificazione di tutti i componenti il Sacro Collegio dei Cardinali in una stessa dignità di Ordine sacro, di episcopale sacramento, e di funzioni altissime nel servizio del governo pontificale in cooperazione al Supremo Gerarca della Santa Chiesa Cattolica Apostolica e Romana» (ivi, 286-290).

${ }^{26}$ Paolo VI, motu proprio Ad purpuratorum patrum, 11 febbraio 1965, in AAS 57 (1965) e in EV, 2, 388-391; in forza degli articoli I e II i patriarchi orientali inseriti nel Collegio cardinalizio sarebbero stati inseriti nell'ordine episcopale, non sarebbero divenuti titolari di una chiesa suburbicaria e neppure sarebbero stati ascritti al clero romano («nec ad clerum Urbis pertinebunt»).

${ }^{27}$ Se n'è occupata recentemente, con ricostruzione archivistica, A. Sammassimo, Cardinalato e collegialità. Codificazione del XX secolo, Milano, Educatt, 2012.

${ }^{28}$ Y. M.-J. Congar, Notes sur le destin de l'idée de collégialité épiscopale en Occident au Moyen Age (VIIe-XVIe siècles), in La collégialité épiscopale, introd. di Y. M.-J. Congar, Parigi, Du Cerf, 1965, spec. p. 118 ss.

${ }^{29}$ Cfr. G. Alberigo, Cardinalato e collegialità, Firenze, Vallecchi, 1969, pp. 187-198. 
«riassorbimento della sua giustificazione ecclesiologica nel suo alveo proprio e autentico, quello della dottrina sull'episcopato e sulla sua natura

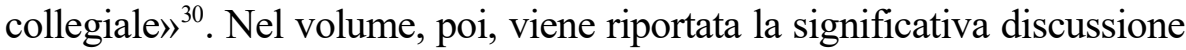
seguita ad un articolo di Karl Rahner che, nel 1963, configurava, per il Collegio cardinalizio, una nuova base di legittimazione, a partire dalla sua funzione elettorale; in tale funzione, il Collegio doveva reputarsi quale «rappresentanza dell'intero episcopato» ${ }^{31}$.

Un lento avvicinamento del Collegio cardinalizio alle ragioni della collegialità episcopale pareva quindi indubbio e l'inserimento di esso tra le manifestazioni della collegialità affettiva divenne, poi, un tema ricorrente anche nell'elaborazione del magistero ${ }^{32}$. Tra tutti gli interventi in tal senso, per ampiezza sembra da ricordare l'omelia che Giovanni Paolo II tenne durante il concistoro del 26 novembre 1994; in essa egli accostava, in una linea di continuità nella collegialità episcopale, collegio dei cardinali e sinodo dei vescovi:

Il Collegio cardinalizio nella sua attuale composizione esprime, assai significativamente, l'unità e l'universalità del popolo di Dio e, soprattutto negli ultimi anni, si è arricchito della crescente presenza di presuli di molte nazioni di ogni Continente: nella schiera dei nuovi cardinali sono rappresentate ben ventiquattro nazioni di ogni parte del mondo. La comunione dell'intero gregge di Dio, nutrito da Cristo, principe dei pastori (cf. Lumen gentium, 6), viene così a rispecchiarsi, in certo modo, nel Collegio cardinalizio, la cui istituzione è molto importante dal punto di vista della tradizione

\footnotetext{
${ }^{30}$ Ivi, p. 211.

${ }^{31}$ Ivi, pp. 201-203.

32 Nella prima intervista dopo l'elezione papa Francesco accomuna, nel principio generale della sinodalità, i concistori e i sinodi: A. Spadaro, Intervista a papa Francesco, in Civiltà cattolica, 2013, 164, p. 458. In un discorso alla Curia romana del 28 giugno 1980, Giovanni Paolo II interponeva il collegio cardinalizio, come espressione della collegialità episcopale, tra il sinodo dei vescovi e le conferenze nazionali dei vescovi: AAS, 72 (1980) 646-647. Più difficile trovare riferimenti al principio di collegialità episcopale nei discorsi che Benedetto XVI rivolgeva ai cardinali in occasione dei concistori per la creazione di cardinali da lui celebrati. Si veda comunque il Discorso del 16 aprile 2007 in Insegnamenti di Benedetto XVI, III, 1, 2007, pp. 678-679.
} 
collegiale della Chiesa. La dimensione collegiale, costitutiva ed essenziale per l'episcopato, trova in effetti una manifestazione eminente ed esemplare nei cardinali stretti intorno al Successore di Pietro. Questa dimensione collegiale, originaria ed intrinseca alla successione apostolica, è andata sviluppandosi nel corso dei secoli, in connessione con la storia della Chiesa e vive oggi un momento particolarmente felice di riscoperta della sua genuinità e di acquisizione di nuove potenzialità. Ciò vale anche per quella sua peculiare espressione che è costituita dal Collegio dei Cardinali: oltre ad abbracciare, per così dire, il mondo intero, esso, grazie alle maggiori possibilità di comunicazione e di incontro, sviluppa oggi in maniera più costante ed efficace il suo servizio. Comunione, collegialità e comunicazione vanno insieme: la comunicazione al servizio della collegialità e la collegialità al servizio della comunione. Singolare espressione della comunione ecclesiale, ed in particolare della collegialità episcopale, è senza dubbio il Sinodo dei Vescovi. Il Concilio Vaticano II, come pure il mio venerato predecessore il Servo di Dio Paolo VI, hanno operato attivamente perché tale istituzione acquistasse sempre più vigore e consistenza. Si è registrato così, negli anni del post-Concilio, un provvidenziale sviluppo della dimensione sinodale della Chiesa, i cui frutti sono sotto gli occhi di tutti. La chiamata alla dignità cardinalizia del Segretario Generale del Sinodo dei Vescovi va vista, oltre che come riconoscimento alla persona, come un'ulteriore tappa di tale incremento. Lo sviluppo della "sinodalità" nella Chiesa, in cui si rispecchia visibilmente la collegialità dell'intero Episcopato, procede di pari passo con la tradizione dei Concistori ordinari e straordinari. Nei sedici anni del mio servizio pastorale, ho avuto occasione di convocare sei Concistori ordinari e cinque straordinari, l'ultimo dei quali ha avuto luogo nel giugno scorso ed è stato dedicato alla preparazione del Grande 
Giubileo dell'Anno 2000. Da quella riunione ho tratto molti elementi per la stesura della Lettera Apostolica Tertio millennio adveniente ${ }^{33}$.

Questo avvicinamento non trovò, però, rilevante spazio nei documenti generali normativi riguardanti direttamente il Collegio dei cardinali. Nel Codex del 1983, anzi, il can. 334 sembra distinguere nettamente l'azione del sinodo, come forma della cooperazione episcopale, da quella dei cardinali, accomunata all'aiuto, offerto al papa, proveniente da «altre persone» ed istituzioni. Nella costituzione apostolica sulla Curia romana, un semplice articolo prospetta l'eventualità di un «concistoro plenario» dei cardinali, disciplinato da una lex propria che mai venne emanata ${ }^{34}$. Nella costituzione apostolica sulla vacanza della Sede apostolica e sull'elezione del papa, poi, non un accenno viene fatto al principio di collegialità episcopale, mentre viene accentuato il carattere romano del Collegio ${ }^{35}$. Vero è ancora che papa Francesco, nel formalizzare con proprio chirografo la struttura e le funzioni di un organismo consultivo già prima insediato, ne attribuiva l'ispirazione all'auspicio, formulato nelle Congregazioni cardinalizie anteriori al conclave del 2013, di creare un «ristretto gruppo di Membri dell'Episcopato» che fosse «espressione della comunione episcopale e dell'ausilio al munus petrinum che l'Episcopato sparso per il mondo può offrire»; il Consiglio era formato da soli cardinali ${ }^{36}$. Lo

\footnotetext{
${ }^{33}$ Giovanni Paolo II, Discorso in occasione del Concistoro per la creazione di 30 nuovi cardinali, 26 novembre 1994, in Insegnamenti di Giovanni Paolo II, XVII, 2, 1994, pp. 883-884.

${ }^{34}$ Giovanni Paolo II, costituzione apostolica Pastor bonus., 28 giugno 1988, in AAS 80 (1988) e in EV, 11, 492-635, art. 23. Per un rapporto di identità tra concistoro straordinario, previsto dal Codex, e concistoro plenario, previsto dalla Costituzione apostolica, v. Arrieta, Diritto dell'organizzazione ecclesiastica, cit., p. 295. Sulle questioni legate alla lex propria del Collegio cardinalizio cfr. A. Sammassimo, De iure condendo: una lex propria per il Collegio cardinalizio, in Jus, 2013, 60, spec. pp. 325-329.

${ }^{35}$ Giovanni Paolo II, costituzione apostolica Universi dominici gregis, 22 febbraio 1996, in AAS 88 (1996) 305-343 e in EV, 15, 98-171.

${ }^{36}$ Francesco, Chirographum quo instituitur Consilium Cardinalium ad adiuvandum Romanum Pontificem in Universali Ecclesia gubernanda adque suscipiendum consilium emendationis Constitutionis Apostolicae «Pastor Bonus» de Curia
} 
stesso (parziale) avvicinamento ha lasciato sospesa una definizione dei confini tra le funzioni consultive del sinodo e quelle del Collegio cardinalizio. Il problema era stato posto dallo stesso Giovanni Paolo II nel discorso del 5 novembre $1979^{37}$.

L'esperienza pluridecennale delle assemblee sinodali, d'altra parte, è stata talvolta oggetto di rilievi critici ${ }^{38}$. Lo stesso papa Francesco, nell'intervista cit., attestando piena considerazione al principio tradizionale canonico delle consultazioni, «reali, non

Romana, 28 settembre 2013, in AAS 105 (2013) 875-876. Sulle implicazioni di tale Chirografo in ordine al rapporto collegio cardinalizio-sinodo, si vedano alcune attente osservazioni in Sammassimo, op. ult. cit., pp. 321-325.

${ }^{37}$ In particolare si veda questo passo: «Sembra dunque che l'incontro del Collegio cardinalizio nell'autunno di quest'anno possa con profitto, occuparsi di un esame, almeno sommario, di alcuni problemi un po' diversi da quelli sui quali lavora il Sinodo dei vescovi. Questi problemi, che in modo introduttivo desidero per lo meno delineare, sono importanti, data la situazione della Chiesa universale, e al tempo stesso sembrano essere più strettamente collegati col ministero del Vescovo di Roma, che non quelli che devono costituire il tema del Sinodo dei vescovi. È ovvio che qui non si può parlare di alcuna rigorosa delimitazione. Subito all'inizio, desidero anche rilevare che, oltre alle questioni che fra poco presenterò da parte mia, conto sulle proposte, che avanzeranno e illustreranno i singoli partecipanti a questo nostro consesso. Prevediamo per questo un dovuto spazio nell'ordine delle nostre sedute. Questo ordine, contrariamente a quanto avviene nel Sinodo dei vescovi, non si basa su alcuno statuto particolare». Giovanni Paolo II, Discorso ai partecipanti alla riunione plenaria del Sacro Collegio dei cardinali, 5 novembre 1979, cit. (v. sopra, nota 59). Sui rapporti tra sinodo e collegio cardinalizio si cfr. Milano, Il sinodo dei vescovi, cit., pp. 371-381.

${ }^{38} \mathrm{Cfr}$., in relazione alla fase storica di realizzazione del sinodo, A. Indelicato, Dal concilio al Sinodo dei vescovi: cronaca di una scelta annunciata?, in A. Melloni-S. Scatena (ed.), Synod and Synodality. Theology, History, Canon Law and Ecumenism in new contact, cit., pp. 237-261, nonché Id., Il sinodo dei vescovi. La collegialità sospesa (1965-1985), cit., spec. pp. 352-361. Si veda anche G. Alberigo, concilio, in G. Barbaglio, G. Bof, S. Dianich (cur.), Teologia (Dizionari San Paolo), cit., pp. 285-287. Per Legrand «attualmente il Sinodo non può gestire né la propria periodicità, né il proprio ordine del giorno, né la pubblicazione delle sue risoluzioni, né la propria composizione (per esempio, il Sinodo speciale per l'America Latina del 1997 comprendeva solo 136 membri che erano stati eletti da loro pari, mentre gli altri 161 erano membri ex officio o designati dal papa)»: Legrand, Le riforme di Francesco, cit., p. 423. 
formali» ${ }^{39}$, aveva modo di esplicitare un pensiero preciso non solo sulla metodologia dei sinodi, ma anche sul principio sinodale nella sua rilevanza generale. Il Papa, in particolare, manifestava l'esigenza che concistori e sinodi vengano resi «meno rigidi nella forma» ${ }^{40}$, aggiungendo come forse sia «il tempo di mutare la metodologia del Sinodo, perché quella attuale mi sembra statica ${ }^{41}$. In questi due contesti sembra che il Papa si riferisca all'interazione tra primato ed episcopato e, dunque, al tema dell'esercizio della collegialità episcopale. Tuttavia egli andava oltre, auspicando una sinodalità «vissuta a vari livelli» e indicandone come attori «la gente, i Vescovi e il Papa ${ }^{42}$. Qui il Papa manifesta notevole interesse per la disciplina dell'Oriente ortodosso, relativamente sia all'esercizio della collegialità episcopale che alla pratica sinodale, traendone un interrogativo per

\footnotetext{
${ }^{39}$ Spadaro, Intervista a papa Francesco, cit., p. 458.

${ }^{40}$ Spadaro, Intervista a papa Francesco, cit., ivi.

${ }^{41}$ Spadaro, Intervista a papa Francesco, cit., p. 466.

${ }^{42}$ Ivi. Si veda anche quanto già affermava il c.d. Documento di Ravenna (13 ottobre 2007) a proposito della nozione di conciliarità: "Il termine conciliarità o sinodalità deriva dalla parola «concilio» (synodos in greco, concilium in latino), che denota soprattutto un raduno di Vescovi che esercitano una particolare responsabilità. Tuttavia, è anche possibile comprendere il termine in un'accezione più ampia, nel senso di tutti i membri della Chiesa (cfr. il vocabolo russo sobornost). [...] «conciliarità» nel suo significato secondo il quale ciascun membro del Corpo di Cristo, in virtù del battesimo, ha il suo spazio e la sua propria responsabilità nella koinônia (communio in latino) eucaristica. La conciliarità riflette il mistero trinitario ed ha il suo fondamento ultimo in tale mistero. Le tre persone della Santa Trinità sono «enumerate», come afferma Basilio il Grande (Sullo Spirito Santo, 45), senza che la designazione come «seconda» o «terza» persona implichi una diminuzione o una subordinazione. Analogamente, esiste anche un ordine tra le Chiese locali, che tuttavia non implica disuguaglianza nella loro natura ecclesiale" (Commissione mista internazionale per il dialogo teologico tra la Chiesa Cattolica Romana e la Chiesa Ortodossa, Documento di Ravenna su "Le conseguenze ecclesiologiche e canoniche della natura sacramentale della Chiesa. Comunione ecclesiale, Conciliarità e Autorità", n. 5, in www.vatican.va).
} 
quanto riguarda l'esercizio del munus petrinum: «Come conciliare in armonia primato petrino e sinodalità?» ${ }^{43}$.

Si è già detto che a tale domanda Papa Francesco ha risposto con la costituzione apostolica Episcopalis communio sul Sinodo dei vescovi del 15 settembre 2018 nella quale, all'art. 1, ha tra l'altro ribadito, come primo aspetto della disciplina di tale organo, che esso è direttamente sottoposto al Romano Pontefice, che ne è il Presidente.

\section{Conclusioni}

Alla luce di quanto avvenuto negli ultimi decenni sembra chiaro come lo sforzo dei Pontefici, da Giovanni Paolo II a Benedetto XVI a Francesco, sia teso non a superare il principio gerarchico che vede i vescovi come «araldi» e «dottori autentici» della fede (Lumen gentium, 25), ma ad integrare tale principio con la pratica della consultazione dei fedeli.

Ci si può interrogare su quale sia il fondamento di tale pratica della consultazione. Lo fece, ad esempio, Georges Chantraine, il quale, chiedendosi se i fedeli condividano la collegialità, dava una

${ }^{43}$ Spadaro, Intervista a papa Francesco, cit., p. 465. Sempre a questo proposito, nel Documento "Sinodalità e Primato nel Primo Millennio: verso una comune comprensione nel servizio all'unità della Chiesa" - firmato a Chieti il 21 settembre 2016 nel corso della quattordicesima sessione plenaria della Commissione mista internazionale per il dialogo teologico tra la Chiesa cattolica e la Chiesa ortodossa si afferma che l'eredità comune di principi teologici, disposizioni canoniche e pratiche liturgiche del Primo Millennio "costituisce un punto di riferimento necessario e una potente fonte d'ispirazione sia per i cattolici sia per gli ortodossi, mentre cercano di curare la ferita della loro divisione all'inizio del terzo millennio. Sulla base di questa eredità comune, entrambi devono riflettere su come il primato, la sinodalità e l'interrelazione che esiste tra loro possono essere concepiti ed esercitati oggi e nel futuro" (Commissione mista internazionale per il dialogo teologico tra la Chiesa Cattolica Romana e la Chiesa Ortodossa, "Sinodalità e primato nel primo millennio”, in: Il Regno-Documenti LXI 2016 17, 576).

$\mathrm{Su}$ tale linea di sviluppo, cfr. da ultimo Saint Irenaeus joint Orthodox-Catholic working group, Servire la comunione: Ripensare il rapporto tra primato $e$ sinodalità, Edizioni Qiqajon, Comunità di Bose, Magnano (Bi) 2019. 
risposta negativa, visto che i fedeli partecipano non del sacerdozio ministeriale, ma del sacerdozio comune ${ }^{44}$. Egli tuttavia, con Eugenio Corecco, ammetteva una analogia tra la corresponsabilità dei fedeli vescovi e quella dei fedeli laici, fondata non sulla collegialità, «forma propria della comunione presso i successori degli Apostoli», né sul potere d'ordine, ma sul potere, comune a tutti i fedeli, di accedere a Dio attraverso la fede e il battesimo. Questo potere è fondamento della comunione e la sinodalità, in tale contesto, può essere definita come la capacità di tutti i fedeli, talvolta il diritto, di essere coinvolti nei momenti sinodali. I fedeli possono esprimere quel consensus, che attesta il sensus fidei $i^{45}$, attraverso questi momenti, o meglio anche attraverso questi momenti ${ }^{46}$, se si presta attenzione alle dinamiche ecclesiali della recezione e della consuetudine ${ }^{47}$.

Il n. 12 della costituzione Lumen gentium fa da sfondo agli istituti partecipativi che consentano un genuino accertamento del senso della fede e bisognerebbe ricordare ancora le parole del

${ }^{44} \mathrm{G}$. Chantraine, Synodalité, expression du sacerdoce commun et du sacerdoce ministériel?, in Nouvelle revue theologique, 1991, 113, p. 357.

${ }^{45}$ Ivi, pp. 357-359.

${ }^{46}$ Sembra significativo il dire che «la sinodalità, al contrario della realtà indicata dal termine sinodo, non è un dato storico, ma una categoria con la quale si indicano alcune proprietà della vita della chiesa a partire dal dato storico che vede, senza mettere in conto i precedenti testimoniati dal libro degli Atti, per lo meno a partire dal II secolo e fino ai nostri giorni, la celebrazione di adunanze finalizzate alla comune decisione su questioni dottrinali e/o disciplinari, sia a livello della singola chiesa locale, sia a livello di più chiese vicine e unite a vari livelli, sia a livello universale di tutta la chiesa»: G. Ruggieri, I sinodi tra storia e teologia, in Associazione Teologica Italiana (ed.), Chiesa e sinodalità. Coscienza, forme, processi, cit., p. 131. Sulla questione del rapporto tra consensus e polo consultivo, cfr. anche P. Tremolada, $L$ 'arte del camminare insieme. Riflessioni sulla sinodalità e il consigliare nella Chiesa, Opera San Francesco di Sales, Brescia 2018.

${ }^{47}$ Sulla recezione si veda, oltre a Y. Congar, La ricezione come realtà ecclesiologica, in Concilium, 1972/7, pp. 75-105, a Tillard, Chiesa di chiese. L'ecclesiologia di comunione, cit., p. 140 ss., anche G. Routhier, La réception d'un concile, Parigi, 1993, pp. 15-65. Sul rapporto tra receptio e consuetudine, M.C. Ruscazio, Receptio legis. Sviluppo storico, profili ecclesiologici, realtà giuridica, Napoli, 2011, pp. 226-240. 
commento, ormai classico, che ne faceva Gérard Philips, il quale, tra l'altro, annotava: «Il senso della fede non è una questione di puro entusiasmo, ma una conoscenza per assimilazione, adattamento, conformità o connaturalità, avrebbe detto san Tommaso. Non può essere confuso con il magistero, né essere assorbito da questo. Al contrario, esso compenetra lo spirito dei credenti per farli vivere nella stessa luce che anima l'autorità docente. In altri termini, il senso della fede è il senso dell'intera Chiesa e non di una delle sue parti, né superiore né inferiore sulla scala gerarchica: è il senso della totalità»; e ancora: «Forse non tutti sanno che, a partire dal Concilio di Trento, $\mathrm{i}$ principali teologi che parlano della credenza universale della comunità non esitano minimamente ad accettare la infallibilitas in credendo; così Melchior Cano, Roberto Bellarmino, Gregorio di Valenza, Suarez, Gonet, Billaurt e i loro continuatori. Di più, nella loro argomentazione essi partono spesso dall'infallibilità della comunità ecclesiale - dunque in credendo - per dedurne l'infallibilità della gerarchia - in docendo. In ciò non vedono alcun pericolo per l'autorità della gerarchia; non esitano neanche a dichiarare che il papa deve tener conto della convinzione unanime dei fedeli» ${ }^{48}$.

Alla luce della tradizione più antica, dunque, nulla di nuovo, dal punto di vista del fondamento, può essere intravisto nello sforzo di attuare una consultazione dei fedeli in materia di dottrina ${ }^{49}$; ne dà testimonianza John Henry Newman in un suo celeberrimo saggio ${ }^{50}$.

\footnotetext{
${ }^{48}$ G. Philips, La Chiesa e il suo mistero. Storia, testo e commento della Lumen gentium, trad. it., Milano, 1975, p. 158.

${ }^{49}$ Nella sua Esortazione apostolica Evangelii gaudium, Papa Francesco dice - e ciò è profondamente rilevante ai fini del nostro discorso - che il vescovo "in alcune circostanze dovrà camminare dietro al popolo [...] soprattutto perché il gregge stesso possiede un suo olfatto per individuare nuove strade" (Francesco, Esortazione apostolica Evangelii Gaudium, n. 31). La metafora dell'olfatto è evidentemente riferita al "sensus fidei fidelium", che è in qualche modo il "fiuto" del Popolo di Dio il quale "non può sbagliarsi nel credere" ("in credendo falli nequit": Lumen gentium, n. 12). Si può vedere una ricostruzione del continuum storico del concetto anche in Commissione Teologica Internazionale, $I l$ sensus fidei nella vita della Chiesa, cit., numeri 22-47.
} 
In realtà, il nuovo modo di procedere sembra, anzitutto, corrispondere all'auspicio che l'ecclesiologia di comunione si inverasse, quanto meno, in momenti di comunicazione ecclesiale interna, se non di strutture adatte allo stesso scopo, così come era stato esplicitato: «L'ecclesiologia della communio non vuole essere una teoria astratta... Essa deve diventare concreta nella vita della chiesa e condurre ivi a un'approfondita comprensione della chiesa $e$ in particolare della liturgia, nonché a una sua forma comunicativa e dialogica rinnovata... La costruzione di una cultura comunicativa, di uno stile dialogale e di strutture comunicative, in particolare sinodali, costituisce un desiderato urgente $\rangle^{51}$. Ancora, nel nuovo modo di procedere sembrano accostati $i$ due principi, gerarchico e comunionale, o, meglio, viene attestato come quest'ultimo si realizzi appieno col mutuo riconoscimento, tra pastori e fedeli, nelle rispettive funzioni, in un «reciproco dare e ricevere» ${ }^{52}$. La consultazione viene realizzata, dunque, senza modalità che in qualche modo potessero far trasparire una qualche contrapposizione tra i fedeli e i pastori, ma attraverso essi e con un metodo per certi versi inedito, esclusa qualunque dialettica tra superiori ed inferiori ${ }^{53}$.

Un primo aspetto di tale metodo sembra realizzare la duplice rappresentanza che il vescovo svolge nella vita ecclesiale; egli, infatti, rappresenta il suo popolo e, presso il popolo, rappresenta l'insieme della Chiesa ${ }^{54}$; anzi, presso il popolo, rappresenta Cristo stesso ${ }^{55}$.

\footnotetext{
${ }^{50}$ J.H. Newman, Sulla consultazione dei fedeli in materia di dottrina, ed. it. a cura di P. Spinucci, Brescia, 1991. Il saggio è citato anche da Chantraine, Synodalité, expression du sacerdoce commun et du sacerdoce ministériel?, cit., p. 359, nota 79.

${ }^{51}$ W. Kasper, Chiesa cattolica. Essenza, realtà, missione, cit., p. 44.

${ }^{52}$ K. Lehmann, L'azione ecclesiale, Milano, 1987, p. 51. Si veda anche quanto schematizzava, già nel suo articolo del 1976, Legrand, Synodes et conseils de l'après-concile, cit., pp. 209-212.

53 Chantraine, Synodalité, expression du sacerdoce commun et du sacerdoce ministériel?, cit., p. 358.

${ }^{54}$ Legrand, Synodes et conseils de l'après-concile, cit., p. 211.

${ }^{55}$ Tillard, Chiesa di chiese. L'ecclesiologia di comunione, cit., p. 225. Cfr. anche O. Condorelli, Sinodalità, consenso, rappresentanza: spunti ricostruttivi nel pensiero
} 
Un ulteriore aspetto, inedito, della consultazione, sta nell'uso di un questionario presinodale reso pubblico e, come tale, facilmente accessibile a chiunque fosse interessato. Durante la presentazione del Documento preparatorio del sinodo straordinario, che si sarebbe poi celebrato nei giorni 5-19 ottobre 2014, il Segretario generale parlava di «rinnovamento metodologico», così che il sinodo risultasse «un vero ed efficace strumento di comunione attraverso il quale si esprima e si realizzi la collegialità auspicata dal concilio Vaticano II ${ }^{56}$. Nella stessa occasione le diocesi, ossia i vescovi, venivano invitati a diffondere il Documento, con l'allegato questionario, «capillarmente nei decanati e nelle parrocchie», e ciò «al fine di ottenere dati concreti e reali sulla tematica sinodale ${ }^{57}$. Nella successiva presentazione (24 giugno 2014) che lo stesso Segretario generale del Sinodo preponeva all'Instrumentum laboris, quanto alle risposte giunte, venivano nominate anzitutto quelle provenienti dai Sinodi delle Chiese Orientali Cattoliche sui iuris, dalle Conferenze episcopali, dai dicasteri della Curia romana e dall'Unione dei Superiori generali. Ma alla Segreteria generale erano arrivate «risposte - dette osservazioni - da un numero significativo di diocesi, parrocchie, movimenti, gruppi, associazioni ecclesiali e realtà familiari, nonché quelle di istituzioni accademiche, specialisti, fedeli ed altri, interessati a far conoscere la propria riflessione ${ }^{58}$. Egualmente, in vista della celebrazione della XIV assemblea generale ordinaria del sinodo, che si è tenuta nei giorni 4-25 ottobre 2015, la Segreteria generale (12 dicembre 2014) trasmetteva il nuovo questionario scegliendo alcuni destinatari istituzionali (Conferenze episcopali, Sinodi delle Chiese Orientali Cattoliche sui

canonistico e teologico medievale (secoli XII-XV), in Repraesentatio. Sinodalità ecclesiale e integrazione politica, cit., pp. 55-61.

${ }^{56}$ Cfr. Il Regno-doc., 2013, 58, p. 695. Su diversi aspetti del mutamento nel metodo sinodale si v. G. Mocellin, Francesco-Sinodo dei vescovi: revisione generale, in Il Regno-att., 2013, 58, pp. 627-628. Cfr. anche D. Vitali, «Un popolo in cammino verso Dio». La sinodalità in Evangelii gaudium, San Paolo, Cinisello balsamo (MI) 2018.

${ }^{57}$ Ivi, p. 696.

${ }^{58}$ Cfr. Supplemento a Il Regno-doc., 2014, 59, p. 444.

$180 \mid$ REVISTA SCIENTIA CANONICA 
iuris, Unione dei Superiori generali, Dicasteri della Curia romana), ma anche fornendo indicazioni per una capillare diffusione di alcuni quesiti sulla recezione della Relatio synodi e «sull'approfondimento dei temi per continuare il cammino sinodale già iniziato ${ }^{59}$.

Papa Francesco, con la decisione di coinvolgere i fedeli in una consultazione sui temi del sinodo, sembra aver voluto innestare sulla pratica della collegialità episcopale un esercizio concreto di corresponsabilità e di partecipazione del popolo dei fedeli. Non pare sia stata esplicitata la qualificazione dottrinale di tale scelta, qualificazione che, d'altra parte, non è detto debba essere univoca. Intendo qui riferirmi alla compiuta ricostruzione di Agostino Montan sulle diverse impostazioni dottrinali poste alla base della corresponsabilità e della partecipazione ecclesiali. Principio di fondamentale eguaglianza dei fedeli, esigenze della communio, pratica della sinodalità, possono essere confluiti nell'adozione del metodo ${ }^{60}$. Questo si è realizzato: in forme allora inedite, diremmo non canoniche in quanto non espressamente regolate dalle norme generali del Codex e da quelle riguardanti l'assemblea generale del sinodo dei vescovi;

\footnotetext{
${ }^{59}$ «Gli Organismi ecclesiali menzionati sono invitati a scegliere le modalità adeguate a tale finalità coinvolgendo tutte le componenti delle chiese particolari ed istituzioni accademiche, organizzazioni, aggregazioni laicali ed altre istanze ecclesiali, allo scopo di promuovere un'ampia consultazione a tutto il Popolo di Dio sulla famiglia secondo l'orientamento del processo sinodale. Una volta conclusa tale consultazione a livello locale ogni Organismo interessato dovrà inviare alla Segreteria Generale del Sinodo dei Vescovi una sintesi dei risultati raggiunti dopo Pasqua, entro il 15 aprile 2015. Pregherei Vostra Eminenza / Eccellenza di distribuire tale Documento alle Diocesi, invitando a diffonderlo subito capillarmente nei decanati e nelle parrocchie al fine di ottenere l'apporto di tutte le componenti del Popolo di Dio per preparare l'Instrumentum laboris»: http://www.vatican.va/roman_curia/synod/documents/rc_synod_doc_20141212_lett era-lineamenta-xiv-assembly_it.html. Si cfr. anche Sinodo de $\bar{i}$ Vescovi, III Assemblea generale straordinaria, Le sfide pastorali sulla famiglia nel contesto dell'Evangelizzazione, Città del Vaticano, 2014, pp. 7-8.

${ }^{60}$ A. Montan, Responsabilità ecclesiale, corresponsabilità e rappresentanza, in Paolo Gherri (ed.), Responsabilità ecclesiale, corresponsabilità e rappresentanza, cit., pp. 14-30.
} 
con modalità tali da non risultare elusivo e, tanto meno, sostitutivo della responsabilità episcopale.

Tali forme sono oggi state codificate proprio da Papa Francesco nella costituzione apostolica Episcopalis communio sul sinodo dei vescovi, a conferma della volontà di rendere sempre più corresponsabile e partecipata la sinodalità nella Chiesa.

\section{Bibliografia}

AAVV, Il sinodo dei vescovi. Natura, metodo, prospettive, a cura di J. Tomko, Città del Vaticano 1985.

AAVV, La Synodalité. La participation au gouvernement dans l'Église (Actes du VIIe congrès international de Droit canonique, Parigi, 21-28 settembre 1990), 2 v., in L'Année canonique, hors série (1992).

Alberigo G., Breve storia del concilio Vaticano II, Bologna, 2005.

Alberigo G., Cardinalato e collegialità, Firenze 1969.

Alberigo G., Conciliarità, futuro delle chiese, in A. Melloni-S. Scatena (ed.), Synod and Synodality. Theology, History, Canon Law and Ecumenism in new contact (International Colloquium Bruges, 2003), (Christianity and history 1), Münster 2005, p. 463.

Alberigo G., Concilio, in G. Barbaglio, G. Bof, S. Dianich (cur.), Teologia (Dizionari San Paolo), pp. 285-287.

Alberigo G., Sinodo come liturgia, in Il Regno-doc., 52 (2007), pp. 443-456.

Arcidiocesi di Milano, La sinodalità nella Chiesa. Un approccio multidisciplinare, Centro Ambrosiano, Milano 2018.

Arrieta J.I., Diritto dell'organizzazione ecclesiastica, Milano, 1997.

Arrieta J.I., El Sínodo de los Obispos, Pamplona 1987.

Arrieta J.I., Il sistema dell'organizzazione ecclesiastica, I ed., Roma 2000.

Associazione Teologica Italiana (ed.), Chiesa e sinodalità. Coscienza, forme, processi, a cura di R. Battocchio e S. Noceti, Milano 2007. 
Associazione Teologica Italiana (ed.), Dossier: Chiesa e sinodalità, a cura di G. Ancona, Bergamo, 2005.

Beinert W., Synode, in J.-Y. Lacoste (dir.), Dictionnaire critique de théologie, p. 1117.

Benedetto XVI, Ordo Synodi Episcoporum del 29 settembre 2006, in AAS 98 (2006), pp. 755-779.

Bettetini A., Formazione della volontà collegiale, principio democratico e verità nel diritto della Chiesa, in Repraesentatio. Sinodalità ecclesiale e integrazione politica, Atti del Convegno di studi organizzato dallo Studio Teologico San Paolo e dalla Facoltà di Giurisprudenza dell'Università degli Studi di Catania, Catania 21-22 aprile 2005, a cura di A. Longhitano, Firenze, 2007, p. 165.

Betti U., La dottrina sull'episcopato nel capitolo III della costituzione dommatica Lumen gentium, Roma 1968.

Bravi M.C., Il sinodo dei vescovi. Istituzione, fini e natura, Roma 1995. Brunini M., In cammino verso la sinodalità ecclesiale sospinti da papa Francesco, in Rassegna di teologia 59 (3/2018), pp. 419-440.

Colombo C., Il compito della teologia, Milano, 1983.

Canobbio G., Libertà di parola e sinodalità tra diritto $e$ responsabilità, Roma 2017.

Chantraine G., Synodalité, expression du sacerdoce commun et $d u$ sacerdoce ministériel?, in Nouvelle revue theologique, 1991, 113, p. 357.

Coda P.- Repole R. (a cura), La sinodalità nella vita e nella missione della Chiesa. Commento a più voci al Documento della Commissione teologica internazionale, Bologna 2019.

Commissione teologica internazionale, Nota Preliminare La sinodalità nella vita e nella missione della Chiesa del 2 marzo 2018, in www.vatican.va.

Condorelli O., Sinodalità, consenso, rappresentanza: spunti ricostruttivi nel pensiero canonistico e teologico medievale (secoli $X I I-X V)$, in Repraesentatio. Sinodalità ecclesiale e integrazione 
politica, Atti del Convegno di studi organizzato dallo Studio Teologico San Paolo e dalla Facoltà di Giurisprudenza dell'Università degli Studi di Catania, Catania 21-22 aprile 2005, a cura di A. Longhitano, Firenze, 2007, pp. 55-61.

Congar Y. M.-J., Notes sur le destin de l'idée de collégialité épiscopale en Occident au Moyen Age (VII $-X V I^{e}$ siècles), in La collégialité épiscopale, introd. di Y. M.-J. Congar, Parigi, Du Cerf, 1965, spec. p. 118 ss.

Congar Y., La ricezione come realtà ecclesiologica, in Concilium, 1972/7, pp. 75-105.

Congregazione per i Vescovi, Pastori di una Chiesa sinodale. Atti del corso annuale di formazione per i nuovi Vescovi, Città del Vaticano 2019.

Corecco E., "Ius et communio». Scritti di diritto canonico, a cura di G. Borgonovo e A. Cattaneo, Casale Monferrato 1997, 2 v.

Corecco E., Ordinatio Fidei, Schriften zum kanonischen Recht, a cura di L. Gerosa e L. Müller, Paderborn 1994.

Eterović N. (ed.), Il sinodo dei vescovi. 40 anni di storia. 1965-2005, Città di Castello (PG) 2005.

Eterović N., Joseph Ratzinger. Benedetto XVI e il sinodo dei vescovi, Città del Vaticano 2014.

Eterović N., Sinodi continentali. I consigli speciali del sinodo dei vescovi, Città del Vaticano 2013.

Fabene F., La sinodalità nella Chiesa e la riforma del Sinodo dei Vescovi, in Commentarium pro religiosis et missionariis 99 (34/2018), pp. 191-202.

Faggioli M., Il vescovo e il Concilio. Modello episcopale e aggiornamento al Vaticano II, Bologna 2005.

Fantappiè C., Chiesa e sinodalità. Per un confronto con Eugenio Corecco, in Ephemerides iuris canonici 58 (2/2018), pp. 461-478.

Francesco, Chirographum quo instituitur Consilium Cardinalium ad adiuvandum Romanum Pontificem in Universali Ecclesia gubernanda 
adque suscipiendum consilium emendationis Constitutionis Apostolicae «Pastor Bonus» de Curia Romana, 28 settembre 2013, in AAS 105 (2013), pp. 875-876.

Francesco, Esortazione apostolica Evangelii Gaudium.

Francesco, Discorso in occasione della Commemorazione del 50.mo anniversario dell'Istituzione del Sinodo dei Vescovi, 17 ottobre 2015, in $A A S 107$ (2015) 1139.

Galavotti E., Jorge Mario Bergoglio e il concilio Vaticano II: Fonte e metodo, in Rivista di teologia dell'evangelizzazione 43 (2018), pp. 61-87.

Gerosa L., Inveramenti istituzionali delle nozioni conciliari di «collegialità»e "partecipazione», in Communio 114 (1990), pp. 76-90.

Giovanni Paolo II, costituzione apostolica Pastor bonus., 28 giugno 1988, in $A A S 80$ (1988).

Giovanni Paolo II, costituzione apostolica Universi dominici gregis, 22 febbraio 1996, in AAS 88 (1996) 305-343.

Giovanni Paolo II, Discorso ai partecipanti alla riunione plenaria del Sacro Collegio dei cardinali, 5 novembre 1979, in Insegnamenti di Giovanni Paolo II, II, 2, 1979, pp. 1060-1069.

Giovanni Paolo II, Discorso in occasione del Concistoro per la creazione di 30 nuovi cardinali, 26 novembre 1994, in Insegnamenti di Giovanni Paolo II, XVII, 2, 1994, pp. 883-884.

Giovanni Paolo II, esortazione apostolica postsinodale Pastores gregis, 16 ottobre 2003, in AAS 96 (2004) pp. 825-924.

Giovanni Paolo II, motu proprio Apostolos Suos, 21 maggio 1998, n. 22, in $A A S 90$ [1998], pp. 641-658.

Giovanni XXIII, motu proprio Cum gravissima, 15 aprile 1962, in AAS 54 (1962), pp. 256-258.

Indelicato A., Il sinodo dei vescovi. La collegialità sospesa (19651985), Bologna 2008.

Institute for Ecumenical Research (ed.), Communio/Koinonia: A New Testament-Early Christian Concept and its Contemporary 
Appropriation and Significance, Strasbourg, Institute for Ecumenical Research, 1990.

Kasper W., Chiesa cattolica. Essenza, realtà, missione, Brescia 2012.

Küng H., Strutture della Chiesa, trad. it. di P. Bernardini-Marzolla, Torino, 1965.

Legrand H., Collégialité, in J.-Y. Lacoste (dir.), Dictionnaire critique de théologie.

Legrand H., La sinodalità al Vaticano II e dopo il Vaticano II. Un'indagine e una riflessione teologica e istituzionale, in Associazione Teologica Italiana (ed.), Chiesa e sinodalità, Milano 2007, pp. 77-82.

Legrand H.-M., Synodes et conseils de l'après-concile, in Nouvelle revue theologique, 98 (1976), pp. 196 ss.

Lehmann K., L'azione ecclesiale, Milano, 1987.

Longhitano A., "Repraesentatio» e partecipazione nell'ordinamento canonico, in Repraesentatio. Sinodalità ecclesiale e integrazione politica, Atti del Convegno di studi organizzato dallo Studio Teologico San Paolo e dalla Facoltà di Giurisprudenza dell'Università degli Studi di Catania, Catania 21-22 aprile 2005, a cura di A. Longhitano, Firenze, 2007, pp. 181-183.

Lüdecke N., Studium Codicis, schola Concilii, Il Regno-att., 2006, 51, pp. 340 ss.

M.G., Le radici del Concilio. Per una teologia della sinodalità, Bologna 2018.

Madrigal Terrazas S. (a cura), La sinodalidad en la vida y en la misión de la Iglesia. Texto y comentario del documento de la Comisión Teológica Internacional, Madrid 2019.

Mandreoli F. (a cura), La teologia di Papa Francesco. Fonti, metodo, orizzonte e conseguenze, Bologna 2019.

Miele M., Dalla sinodalità alla collegialità nella codificazione latina, Padova 2004. 
Miele M., Papa Francesco e gli sviluppi recenti del metodo sinodale, in O. Fumagalli Carulli - A. Sammassimo, Famiglia e matrimonio di fronte al Sinodo. Il punto di vista dei giuristi, Milano 2015, pp. 317-346.

Milano G.P., Il sinodo dei vescovi, Milano 1985.

Moda A., Sulla sinodalità. Per un percorso bibliografico, in Associazione Teologica Italiana (ed.), Dossier: Chiesa e sinodalità, cit., pp. 205-329.

Montan A., Responsabilità ecclesiale, corresponsabilità e rappresentanza, in Paolo Gherri (ed.), Responsabilità ecclesiale, corresponsabilità e rappresentanza (Atti della quarta giornata canonistica interdisciplinare), Roma, 2010, pp. 14-30.

Musselli L., Forme ed Istituti di rappresentanza canonica, in Paolo Gherri (ed.), Responsabilità ecclesiale, corresponsabilità e rappresentanza (Atti della quarta giornata canonistica interdisciplinare), Roma, 2010, pp. 110-112.

Newman J.H., Sulla consultazione dei fedeli in materia di dottrina, ed. it. a cura di P. Spinucci, Brescia, 1991.

Paolo VI, motu proprio Ad purpuratorum patrum, 11 febbraio 1965, in $A A S 57$ (1965).

Paolo VI, motu proprio Apostolica sollicitudo, 15 settembre 1965, in AAS 57 (1965).

Philips G., La Chiesa e il suo mistero. Storia, testo e commento della Lumen gentium, trad. it., Milano, 1975.

Ratzinger J., Chiesa, ecumenismo e politica. Nuovi saggi di ecclesiologia, trad. it., Milano 1987.

Remossi A., Il concetto di rappresentatività nell'ordinamento canonico, in Periodica de re canonica, 2005, 94, pp. 604-607.

Routhier G., La réception d'un concile, Parigi, 1993, pp. 15-65.

Routhier G., Perspectives et dimensions d'une recherche sur la synodalité de l'Église, in A. Melloni-S. Scatena (ed.), Synod and Synodality, Münster 2005, pp. 91-94. 
Ruscazio M.C., Receptio legis. Sviluppo storico, profili ecclesiologici, realtà giuridica, Napoli 2011.

Sammassimo A., Cardinalato e collegialità. Codificazione del XX secolo, Milano 2012.

Sammassimo A., De iure condendo: una lex propria per il Collegio cardinalizio, in Jus, 2013, 60, spec. pp. 325-329.

Sinodo dei Vescovi, III Assemblea generale straordinaria, Le sfide pastorali sulla famiglia nel contesto dell'Evangelizzazione

Spadaro A., Intervista a papa Francesco, in Civiltà cattolica, 2013, 164, p. 458.

Tagle L.A.G., La tempesta di novembre: la «settimana nera», in G. Alberigo (dir.), Storia del concilio Vaticano II, IV, Bologna, 1999, pp. 446-475.

Tillard J.M., Chiesa di chiese. L'ecclesiologia di comunione, trad. it., Brescia, 1989

Tillard J.M., Chiesa di chiese. L'ecclesiologia di comunione, cit., pp. 140 ss.

Tremolada P., L'arte del camminare insieme. Riflessioni sulla sinodalità e il consigliare nella Chiesa, Brescia 2018.

Vitali D., «Un popolo in cammino verso Dio». La sinodalità in Evangelii gaudium, Cinisello Balsamo (MI) 2018.

Zambon G., Riconoscimento reciproco di soggettività tra laici e ministri ordinati in ordine ad una forma sinodale di chiesa, in Associazione Teologica Italiana (ed.), Chiesa e sinodalità. Coscienza, forme, processi, Milano 2007. 